\title{
Deep Disagreement and Patience as an Argumentative Virtue
}

\author{
KATHRYN Phillips
}

\author{
Writing, Speaking, and Argument Program \\ University of Rochester \\ PO Box 270058, Rochester, NY 14627 \\ USA \\ kathryn.phillips@rochester.edu
}

\begin{abstract}
During a year when there is much tumult around the world and in the United States in particular, it might be surprising to encounter a paper about patience and argumentation. In this paper, I explore the notion of deep disagreement, with an eye to moral and political contexts in particular, in order to motivate the idea that patience is an argumentative virtue that we ought to cultivate. This is particularly so because of the extended nature of argumentation and the slow rate at which we change our minds. I raise a concern about how calls for patience have been misused in the past and argue that if we accept patience as an argumentative virtue, we should hold people in positions of power, in particular, to account.
\end{abstract}

Résumé: Pendant une année tumultueuse dans le monde et aux ÉtatsUnis en particulier, la publication d'un article sur la patience et l'argumentation peut paraître surprenante. Dans cet article, j'explore la notion de désaccord profond, avec une attention sur les contextes moraux et politiques afin d'avancer l'idée que la patience est une vertu argumentative que nous devons cultiver. Cela est particulièrement vrai en raison de la nature de l'argumentation et de la lenteur avec laquelle nous changeons d'avis. Je m'inquiète de la façon dont les appels à la patience ont été mal utilisés dans le passé et je soutiens que si nous acceptons la patience comme une vertu argumentative, nous devrions en particulier obliger les personnes en position de pouvoir à rendre des comptes.

Keywords: argumentative virtue, deep disagreement, patience. 


\section{Introduction}

When I first started writing this paper in early 2020, the United States was at the beginning of a presidential election year, and the heated Democratic party primaries were wrapping up. In February, Elizabeth Warren and Tulsi Gabbard became the last two women to drop out of the race, leaving only two white men (Bernie Sanders and eventual nominee and later President Joe Biden), albeit with deep ideological distinctions, contending for the nomination. At that time, I found myself in what felt like deep disagreements with friends about the role sexism plays in how we evaluate candidates and who Americans tend to see as fit for our highest political office. Soon after, COVID-19 was confirmed to have spread to the United States, and we entered lockdown to slow the spread. There was a great deal that we did not know, and at the time of writing, there is still much we do not know, but in the face of the pandemic, the United States continues to struggle with partisanship, distrust, and now politicization of public health efforts.

It was also in February that Ahmaud Abery was murdered by two white men while out jogging in Georgia (Fausset 2021). In March, Breonna Taylor was murdered by police after a no knock warrant in Louisville, KY. In May, George Floyd was murdered by a Minneapolis police officer, which was followed by a surge in protests demanding racial justice. Systemic racism and white supremacy continue to take lives every day, while many Americans remain unwilling or unable to even admit we have a problem. While Joe Biden has clearly won the presidential election, and while that win is decisive, it is still a narrower win than many of us would have liked to have seen against an openly racist president who has repeatedly refused to denounce white supremacy (Lemire et al. 2020) and more recently played a role in inciting a riot and fomenting anti-democratic violence culminating in a mob storming the Capitol (Woodward and Riechmann 2021)

Given these circumstances in the United States in which trust in each other is low, injustice is high, and all the while we are battling a global pandemic, patience might seem like a low priority, or worse. The concept of patience has been misused to advocate for enduring injustice and to wait until tempers calm rather than affirming the role of emotions in deliberations and calls for 
change. While patience may seem like a strange focus at this moment, our current circumstances make it a particularly compelling time to talk about deep disagreement — cases in which some have argued that no rational resolution is available - and I will argue that patience has an important role to play when we encounter deep disagreements. Given the prevalence of distrust and injustice, it is important to think both about how epistemic gulfs form between us that make argumentation seem out of reach, and also what tools are available to help us continue to seek resolution through argumentation, rather than other more coercive means. Tracy Bowell argues elsewhere in this volume that the challenges of our current communicative landscape make virtue argumentation particularly appealing because it allows us to focus on broader goals of argumentation, such as understanding, rather than just truth and falsity. With a focus on cultivating the character traits necessary to be a good arguer, a virtue-theoretic approach to argumentation can help us better understand our individual and collective failings that might render argumentation impossible, particularly when we are perpetuating argumentative (and moral) vice through silencing, erasing, and other forms of oppression.

In this paper, I will focus on deep disagreements in the moral and political realms both because of their importance, and also their susceptibility to becoming deep. More specifically, I argue that deep disagreements help us see that patience is an important argumentative virtue given the extended nature of argumentation that is particularly obvious when it comes to moral and political debate. Moral and political disagreements help us see that minds do not change quickly and that developing and maintaining trust is central to continued engagement. This requires awareness of our own privilege, experiences, contextual knowledge, and biases as well as a commitment to trying to better understand each other. My central claim is that cultivating the virtue of patience is necessary for creating these felicitous argumentative conditions. While I argue for the importance of patience as an argumentative virtue, particularly in the face of moral and political disagreements that become deep, I will simultaneously explore a major hesitancy I have about the role of patience in argumentation. My concerns arise primarily from the prevalence of argumentative and epistem- 
ic injustice, which results in a disproportionate distribution of demands for patience to those who have the least reason to be patient, and the absence of patience in individuals who need to cultivate it the most.

My confidence that patience is an important argumentative virtue combined with my concern about that very same claim is best explained by divisions between ideal and non-ideal theory. ${ }^{1}$ When developing an ideal account of argumentation, I argue that patience is unambiguously an argumentative virtue because it is necessary for productive engagement, deliberation, continuation of discussion, and the possibility of collaborative resolution. From a non-ideal perspective, the story is much more complicated by the ways in which patience is distributed unevenly and the role of power dynamics in determining who is indeed patient and who tends to fail to develop the virtue. Put another way, normatively, we need patience to continue to argue with one another, understand more, and learn things that we wouldn't if we refused to encounter or continue arguing with people that we disagree with. However, this burden is immensely disproportionately distributed to those with less power when we look at argumentative situations descriptively rather than normatively.

In the next section of this paper, I will give an overview of the literature on deep disagreement and why I take moral and political issues to be particularly susceptible to becoming deep. The third section includes a brief discussion of virtue argumentation before I turn my attention to the importance of patience as an argumentative virtue in ideal cases. The fourth section explores the problems for patience from a non-ideal perspective, and I conclude with some tentative ideas about argumentative strategies that we might develop to redistribute the burden of patience.

\footnotetext{
${ }^{1}$ The distinction may be more familiar in moral and political philosophy, where theorists try to explain gaps between normative ideals and the descriptive reality of our actions, but this distinction is becoming more prevalent in other domains, such as philosophy of language - see, for instance, Cappelen and Dever's Bad Language.
} 


\section{Deep disagreement}

There are competing accounts of the conditions that constitute an authentically deep disagreement, how they might be resolvable, and also a variety of concepts that are similar to, though distinct from, deep disagreement as it was initially defined, such as persistent (Amenábar 2018) or recalcitrant disagreements (Kloster 2018). The concept of deep disagreement originated in Robert Fogelin's 1985, "The Logic of Deep Disagreements," where he introduced the phrase to pick out a particular class of disputesthose that are rationally irresolvable: "there are disagreements, sometimes on important issues, which by their nature, are not subject to rational resolution" (Fogelin, 1985, p. 7). According to Fogelin, disagreements can be tense without being deep, or irresolvable given the shortcomings of one or both participants without being deep, but deep disagreements are not resolvable even though all participants are rational because clashing framework propositions leave the interlocutors with only (non-rational) persuasive means to get out of the disagreement. In what follows, I'll first give an overview of some debates about the nature of deep disagreement, followed by a discussion of what kinds of deep disagreements I'm most interested in here and why I think they're so challenging to resolve.

In the years since Fogelin's introduction of deep disagreement, the discussion has expanded beyond the possibility of rational resolution to investigations of broader issues, such as what it means to share a framework (Davson-Galle 1992), testing the limits of the concept of rational persuasion (Lugg 1986), levels of disagreement (Duran 2016), and developing thoughtful accounts of what kind of repair might need to take place in order to mitigate the depth of the disagreement (Kloster 2018). Some of this work aims at delimitating cases in which rational persuasion must give way to conversion or some other non-rational process if agreement is to be reached, while another primary area of focus is distinguishing hard problems from those disagreements that are genuinely irresolvable.

One challenge of analyzing the notion of deep disagreement is distinguishing between accounts that are fully ideal and meant to help us theorize versus understandings that are meant to be practi- 
cally applicable and focus on non-ideal argument. The ideal notion tends to be more aligned with traditional epistemological debates about disagreement, and asks us to understand abstract, ideal epistemic peers with identical evidence and perfect trust who nonetheless disagree. On this understanding of deep disagreement, the rational irresolvability is analytic, rather than based on any contextual factors. This makes the possibility of resolution impossible unless one takes the position that framework propositions are just more evidence to be argued over. Scott Aikin calls such disagreement "absolutely deep" because he takes depth, like Duran, to be a gradable notion, while arguing that Foeglin-style deep disagreements exclude rational resolution by definition (Aikin 2020). Non-ideal accounts make more room for contextual factors to explain the disagreement and help us understand a richer set of possibilities regarding how such disagreements might be addressed. This distinction has emerged through deliberations about the extent to which we ought to be optimistic or pessimistic about the possibility of resolving deep disagreements.

An example of denying that deep disagreements are irresolvable even if one approaches deep disagreement from an ideal perspective comes from Richard Feldman (2005). He largely denies the possibility of deep disagreement given his optimism about the possibility of rational resolution. Feldman's account draws a strong parallel between a mundane disagreement about nonframework propositions, such as who won the 1955 World Series. He assumes a situation in which each of the interlocutors has a reputable source that gives different information, and in this case, the rational resolution is to suspend judgment given the conflicting evidence. According to Feldman, practical considerations such as interest in the outcome ought to be stripped away in order to give a purely epistemic assessment. Feldman goes on to argue that the underlying principles, or framework propositions, are just more evidence to consider, and doesn't see any reason why rational resolutions to framework propositions might be more complicated than disagreements about other sorts of claims. Even if they are, suspension of judgment is available as a rational resolution.

An example of a more non-ideal approach to understanding deep disagreement can be found in Kloster (2018). Kloster identi- 
fies two central factors that might be disputed in instances of deep disagreement: 1) relevant shared background beliefs and 2) a procedure for negotiating disagreement that includes affective and social factors such as power dynamics shaping responses like fear. Her account explicitly questions an overly idealized model of deep disagreement given the manner in which conceptions of the ideal reasoner can be shaped by gender, class, and racial bias. To account for affective and social factors, Kloster expands on the notion of deep disagreement to include recalcitrant, rationally irresolvable disagreements whose irresolvability stems from social conditions such as lack of trust (Kloster 2018).

Similar to Kloster, Scott Aikin favors a non-ideal approach to deep disagreement, given his skepticism that absolutely deep disagreements are anything more than a theoretical possibility (Aikin 2020). Similar to Claudio Duran, Aikin argues that we should understand disagreement as having a variety of levels of depth (Aikin 2019). This means that rather than there existing a clear boundary between deep disagreements and regular disagreements, they run along a continuum. Aikin's account suggests that we start by arguing about an issue, but when we come to understand arguments as deeper than the particular issue, then we move to reasoning about each other. This second move is an attempt to understand how the other person got to the place they are in their beliefs because the disagreement is so large that we turn to wondering about each other, and also potentially closing ourselves off to continued discussion. While Aikin might intend this as a purely descriptive claim, this is perhaps a strategy we should employ to better understand what is grounding the values or other framework propositions that are making rational engagement more difficult.

I am primarily interested in non-ideal notions of deep disagreement because, like Aikin, and perhaps Feldman, I am skeptical that absolutely deep disagreements are anything more than a theoretical possibility. Some of the most interesting cases of deep disagreements are cases of moral and political disagreement. These are likely often best understood as recalcitrant disagreements or disagreements of depth that are not absolutely deep. Moral and political disagreements are also particularly interesting because they are more resistant to Feldman's prescription of sus- 
pension of belief given their practical focus. It is interesting to note that the two examples Fogelin gave us in his paper are moral disputes: disagreements about abortion and affirmative action.

The susceptibility of moral disagreements to becoming deep might be explained in a variety of ways. One possibility is the apparent prevalence of a naïve subjective view of morality that suggests everyone just has their own moral preferences and they cannot be debated given the subjective nature of such beliefs. This could leave disagreements deep because of an inability to question moral commitments. Another possibility as to why moral disputes might be particularly ripe for deep disagreement comes from the opposite metaethical view that robust realism demands that we hold our ground on moral claims. David Enoch has argued that moral claims are actually nothing like disagreements about preferences such as which ice cream to buy or what we have for lunch today. Specifically, Enoch claims:

Had morality not been objective - had it been, for instance, preference-based - we would have been required to behave in the face of conflicts based on moral disagreements as we are required to behave in the face of conflicts based on mere preferences. That is, we would have been required to step back, go impartial, view our own commitments as just the commitments of one party among others, and compromise. But - and this is a substantive moral premise - we are not required so to behave in the face of moral disagreement and conflict (Enoch 2014).

At the same time, deep disagreements seem just as prevalent in political contexts as they are in moral contexts, and the deeply personal nature of such commitments sets them up as central arenas for deep disagreement.

If deep disagreement is particularly salient in moral and political spheres, this suggests a connection between deep disagreements and the related notions of transformative experience and aspiration. Both transformative experience and aspiration seem relevant to the domain of deep disagreement because they raise questions about whether intrapersonal experiences can be rational in ways that mirror some of the interpersonal dynamics of deep disagreement. This analogous framework can help us better under- 
stand the notions of deep disagreement and also to highlight the ways that framework propositions are likely deeply tied to an individual's sense of self.

The notion of transformative experience was developed by Laurie Paul to investigate the rationality of making choices that will transform a person's subjective experience in ways that are unknowable prior to having the experience. According to Paul, "a transformative experience is a kind of experience that is both radically new to the agent and changes her in a deep and fundamental way; there are experiences such as becoming a parent, discovering a new faith, emigrating to a new country, or fighting in a war. Such experiences can be both epistemically and personally transformative" (Paul 2015, p. 761). Paul concludes that in the case of transformative experiences, one can have a rationally accessible higher-order desire to choose a radically novel and transformative experience, but they cannot rationally choose an unknowable first-order desire such as becoming a parent, converting to a new religion, etc.

Several challenges have been posed to Paul's conclusion that transformative experiences cannot be rationally chosen. Veronica Ivy, for instance, argues that in certain cases, such as gender transitions, individuals can know the expected utility of not choosing the transformative experience, even if one cannot know what it will be like to undergo the transformative experience (McKinnon 2015). This creates some basis for rationality in decision making even if one cannot know the expected utility of the transformative experience. Agnes Callard goes a bit further in developing her model of aspiration, which rejects some aspects of Paul's account of transformative experience. For Callard, such transformations are a process of self-cultivation and transformation of values that transforms the ethical self. The ethical self is understood as follows: "This self is composed of those features of a person that have ethical significance - they're the features in virtue of which you are praise- or blameworthy, beloved or hated... Which features of a person have ethical significance? They will differ from person to person, and they depend at least in part on what the person takes to have ethical significance" (Callard 2018, p. 32). For Callard, this is a slow process that presents a unique form of rationality- 
one where an agent moves slowly towards what she aspires to be: "Aspiration is rational, purposive value-acquisition" (Callard 2018 , p. 8). In both cases, we see an absence of important kinds of experience, which may mirror some of the challenges we see in deep disagreement. Similarly, Paul, Ivy, and Callard disagree on the extent to which certain kinds of decisions, namely those that transform the self, can be rational. This has much in common with debates about the possibility of rational resolution with respect to deep disagreement.

In cases of deep disagreement, it might also be the case that there is the possibility of transformation should framework propositions change. That would suggest that those engaged in deep disagreements might have something more like a sense of self at stake, at least in the moral and political domains, which may account in part for why such disagreements are so difficult to resolve. If such disagreements are rationally resolvable, it seems to require developing common ground and enough shared commitments to continue to argue, which will likely take place over a very long period of time-in many cases, years of continued friendship, continued education, and exposure to various forms of arguments about the target issues. Argumentation that proceeds slowly and is particularly challenging makes space to explore patience as an argumentative virtue.

\section{Argumentative virtue and patience}

Virtue argumentation is structurally similar to virtue theories in ethics and epistemology that have moved away from attempts to formulate universal, abstract principles in favor of focusing on the cultivation of virtues. Virtues are typically understood as excellences of character and are the primary concept in the relevant domain. In ethics, this usually means that the good action is subordinate to the good actor, and in argumentation theory, we might understand the argument as secondary to the arguer. Andrew Aberdein offers a clear overview of major movements in virtue ethics from ancient Greek thought through a Christian turn and more modern revivals, as well as the somewhat more complicated relationship virtues might have with epistemological concepts such 
as knowledge and justification: "They have been represented variously as possessing conceptual priority over the traditional concepts, or as explanatorily but not conceptually prior, or merely as a reliable guide" (Aberdein, 2010, p.166). In all domains, the conceptual priority is somewhat complicated; for instance, in ethics, one major objection is the extent to which a virtue ethics is action-guiding, and similarly in argumentation theory, one major concern is the extent to which virtue argumentation conflicts with concerns about ad hominem attacks (for instance, see the thoughtful discussion in [Bowell and Kingsbury 2013]).

Virtue argumentation has a natural affinity with rhetorical and dialectical models of argumentation that both, to different extents, conceptualize argumentation as a procedure or process rather than having a strict focus on argument as a product. This, to some extent, begins to respond to concerns about ad hominem and virtue argumentation because these broad frameworks suggest that argument is inextricable in some sense from the context and procedures used to develop the argument in question. In addition to understanding argumentation as process rather than just product, in rhetorical and dialectical models of argumentation, we see more focus on the arguers, rather than just the argument. In dialectical models of argumentation, there is a focus on the moves arguers should make, while rhetoric often has a strong focus on the situated nature of speakers and audiences, taking a more descriptive and less normative approach to understanding argumentation. As we saw in Kloster and also Aikin above, in the cases of suspected deep disagreement, one potential strategy includes a pivot towards the person and/or social context and away from the argument in order to create space for resolution. These possibilities for resolution both push us towards virtue argumentation because of the importance of understanding the role of character, as well as the role a virtuous arguer may play in helping construct more felicitous conditions for argumentation.

A variety of scholars have laid out potential taxonomies for deliberative (Aikin and Clanton 2010) or argumentative virtues and vices. Daniel Cohen suggested that willingness to engage, listen, modify one's position, and question the obvious are argumentative virtues that facilitate the process of reason-giving in the 
service of raising the credibility of the conclusion (Cohen 2005). Aberdein builds on Cohen's initial four core virtues and develops a typology of argumentative virtues and vices by situating the virtues as the mean between two vicious extremes. In this way, willingness to engage in argumentation is situated between being uncommunicative or mistrusting reason at one extreme and being intellectually rash and overzealous at the other extreme (Aberdein 2016). Aikin and Clanton argue that (deliberative) wit, friendliness, temperance, courage, sincerity, and humility facilitate the development of knowledge (Aikin and Clanton 2010). All of these authors suggest that their lists are incomplete and meant to be built upon, and I argue that we should add the virtue of patience to these lists because argumentation is an activity that takes place over time, sometimes quite a long time. When it comes to recalcitrant or deep disagreements in particular, it takes long periods of time to change minds about particularly important and contentious issues (for more thoughtful discussion on how people change their minds, see [Kjeldsen 2020]).

Aristotelian virtues are understood to be defined relative to particular spheres where the virtue represents the excellent mean between two extremes and the extremes delineate the related vices. As we saw above, Kloster argued that when social trust is absent, this deepens disagreement. Along similar lines, Cohen and Miller (2016) suggest that ideal arguments feature cognitive compathy, which is understood as "a phenomenon in ideal arguments when arguers are on the same page, genuinely engaging, doing it welland doing it together" (Cohen and Miller 2016). The relevant form of cognitive sharing that occurs when cognitive compathy is present is best facilitated, according to these authors, by cultivating the virtue of open-mindedness, but is also dependent on the "subject matter, the context, and the personal chemistry of the arguers" (Cohen and Miller 2016). This suggests that virtues that foster group cohesion and felicitous social conditions are important for argumentation, as well as virtues of self-control that are conducive to those social conditions. Virtues of self-control are often understood to be virtues such as courage, temperance, and patience. Aberdein (2019) has also argued previously that courage is partic- 
ularly salient when it comes to deep disagreement, though little has been said about the importance of patience.

Denise Vigani argues that in order to understand patience as an Aristotelian virtue, we first need to identify the relevant sphere in which it operates so that we can pick out the virtuous mean and vicious extremes. According to Vigani, patience, like temperance and courage, is a virtue of self-control and is conceptually linked with waiting, enduring, persevering, and tolerating - all capacities that have strong temporal elements (Vigani 2017). She concludes that patience is best understood as a virtue whose domain is time - it is the excellence between hastiness and sluggishness.

In practical domains, we might understand the patient person as the one who takes the right action at the right time or the person whose desires are under control, such the patient person who continues the appropriate pursuits in frustrating circumstances. In the intellectual domain of argumentation, one way we can understand the hasty arguer is to imagine one all too willing to engage in argumentation without attempts to understand their interlocutor (including their motivation, character, background beliefs, and experience) or the social context and how it shapes the present disagreement. Similarly, the hasty arguer might not take the time to appreciate the power dynamics within the argumentative exchange because she may be so focused on argument as product, getting to the answer as quickly as possible, or even may be hasty because of her own desire to win the argument rather than work through its complexity with her interlocutors. The sluggish arguer may be understood as one who backs off completely or fails to ever take a position, perhaps even because of an admirable concern that there is always more to learn, which leads her to a great deal of uncertainty and an unwillingness to engage. Another possibility is that though the sluggish arguer has plenty of arguments she could make, she fails to make them for a variety of other reasons.

In some ways, this aligns with Aberdein and Cohen's primary virtues and vices that revolved around willingness to engage. Patience, however, goes further, because the arguer who cultivates patience develops means to continue to engage over time, and given her appreciation of the extended nature of argumentation 
and the ways that new ideas sometimes take days, weeks, or years to settle in, she avoids frustrations that might cause her to disengage (or at least is able to manage them). Furthermore, the patient arguer is able to take the time to think expansively about her interlocutors and the relevant social context in order to identify (over time and through engagement) ways to change the argumentative conditions in order to create space for argumentation. In the case of deep disagreement, this might include pivoting towards understanding the interlocutor's character, experiences, and framework beliefs to better understand the nature of the disagreement. This might also include working to understand how power dynamics might be facilitating or thwarting continued discussion. Furthermore, the patient arguer, recognizing the temporally extended nature of argumentation, will be better able to recognize and accept their own weaknesses in ways that will facilitate the continued engagement of their interlocutors.

A more specific example of how patience can help us address the complex nature of deep disagreement comes from a concern raised by Chris Campolo. He argues that under certain conditions, such as deep disagreement, it becomes irresponsible to continue to engage with interlocutors and that we have not just an epistemic but also a moral duty not to pursue continued reasoning in the case of deep disagreements (Campolo 2019). He believes that there is a danger that we will degrade our reasoning ability by pretending to draw nearer to consensus with the other person when there is a real gap between our interests and values that underlie the reasoning process. According to Campolo: "What we ought to do when we find that we may deeply disagree, is to stop reasoning, and then, if going on together is important, see if we can make substantive changes in what one or all of us understand. This is a slow and painstaking process" (Campolo, p. 722). Campolo, contra some of the other more optimistic views about deep disagreement, is concerned about the consequences of continued engagement in argumentation at a certain level of depth.

The disagreement between Campolo and optimists about deep disagreement regarding whether we ought to continue to engage makes room for considering patience as a way to open up possibilities for continued engagement by developing the tendency to see 
argument as multifaceted with many goals, dependent on social context, and shaped by speakers and audience. Taking time can give shape to other elements of the argumentative process that are necessary for rational persuasion to occur, even if there is disagreement about whether these conditions are part of, or outside of, rationality itself. The patient arguer can consider many possibilities, take breaks when she needs it, and appreciate that argumentation, especially about hard problems, is a long-term pursuit. The patient arguer can negotiate when it is productive for her to continue to engage, and how, and also when it is not, as well as taking the time to navigate productive ways to repair argumentative situations. This provides a sort of middle ground between Campolo's concerns and more optimistic approaches. Understanding patience as an important virtue when it comes to deep disagreement provides more options than just continuing to engage or not because the primary domain of patience is time, and when it comes to deep disagreement in particular, understanding the extended nature of argumentation across time is particularly crucial.

\section{Some problems with patience}

While its temporal domain provides promise for patience as an argumentative virtue, authors who have written about patience in the moral and epistemic domains are aware of historical and descriptive concerns about patience. These concerns are often gendered; for instance, Jason Kawall says: "We might imagine a woman in an abusive marriage who 'patiently' endures the situation" (Kawall, 2016, p. 4). Eamon Callan extends the concern to victims of exploitation more generally:

There are psychological traits that increase the ease with which we can be mistreated by others, and these may be extolled as virtues by those who would do the mistreating, palliate its evil or deny its avoidability. Patience can ensure compliance among the victims of exploitation, and so, unsurprisingly, it has often been recommended to the poor or to women as a virtue that befits their station and its duties. (Callan 1993, p. 538) 
Referencing Callan's example of women and the poor, Vigani suggests that passivity, which is often mistaken for patience, may be endorsed by particular individuals as "a result of upbringing, misplaced loyalty, or other reasons" (Vigani 2017, p. 334). All authors seem to acknowledge that in theory as well as practice, "patience" is used in problematic ways, such as in service of silencing.

Vigani and Callan respond in similar ways, appealing to the nature of the virtue rather than colloquial conceptions of patience. Vigani explicitly calls her account of patience a thin account because it relies on "granting the appropriate amount of time" - the thinness comes from the need to fill out what "appropriate" means here. Callan similarly says: "It is only a puerile, coarse-grained patience that could motivate a blanket impassivity toward evils that are fit objects of indignant resistance. One cannot reasonably argue against the ethical centrality of patience by dwelling on the deficiencies of its least discriminating versions any more than one can make a decent case for the marginalization of courage merely by noting the moral hazards of a naive bravery" (Callan 1993, p. 539). In other words, these oppressive uses of "patience" aren't the virtue of patience at all.

These responses, as Vigani suggests noting the thinness of the account, show that analyses of patience need more detail and substance to distinguish colloquial accounts of patience, which mistake sluggishness or even use calls for "patience" as mechanisms of systemic oppression, from the virtue itself. This means that patience is a concept in need of repair. A related concern is that conceptualizing patience as a virtue can fail to engage with the question of how patience ought to be conceptualized and cultivated in our non-ideal world of actual argumentation. Generally speaking, in our actual world, those who are often required to cultivate the most patience in order to engage in argumentation are also those who are most likely to face epistemic harms, such as testimonial and hermeneutic injustice.

The notion of "epistemic injustice" was popularized by Miranda Fricker in her 2007 book of the same name. Testimonial injustice, according to Fricker, occurs when knowers face credibility deficits based on identity prejudices, which leads hearers to 
ascribe less weight to their testimony on the basis of epistemically irrelevant factors such as race, gender, ability, and so on. She argues that while there are practical harms in unjustly assigning credibility deficits, it is also a harm to persons specifically as knowers. Fricker acknowledges that credibility excesses are also possible, but she focuses on deficits as particularly unjust. This is one way that we can see that patience becomes unevenly distributed - those unjustly discriminated against and whose testimony is systematically devalued are asked in practice to do the work of combatting those identity prejudices by demonstrating their value rather than having it assumed.

Patrick Bondy argues that in addition to epistemic injustice, we ought to understand argumentative injustice as a unique phenomenon (Bondy 2010). Bondy takes argumentative injustice to be distinct from its epistemic cousin because it operates both in the form of deficiency and excess, contra Fricker's claim that epistemic injustice is mainly an issue of credibility deficits that are ascribed based on a false identity stereotype. Granting too little credibility to agents as arguers harms the participants in argumentative exchanges through undermining the rationality of the argumentation, distorting the assessment of the arguer who is being prejudicially assessed. Deficits can also damage the ability of the falsely stereotyped agent to engage in argumentation. Excesses, according to Bondy, have three negative consequences on the participants: they can create an overly narrow understanding of the relevant reasons, make agents think they are more capable arguers than they are, and prevent them from being adequately challenged and thus more able to see and engage with the relevant reasons. These excesses and deficiencies can cultivate arguers that have the vices of sluggishness and hastiness.

Hermeneutic injustice is another form of epistemic injustice that occurs due to a lack of adequate conceptual resources for oppressed groups to effectively communicate their experience to others and even to themselves (Fricker 2007). Charlie Crerar has argued more recently for a broader understanding of hermeneutic injustice that extends beyond a lack of conceptual resources given the oppressive social situation to situations in which conceptual resources are available but the relevant parties are silenced none- 
theless (Crerar 2016). Crerar's focus is on taboos. In these cases, there are perfectly adequate conceptual resources, but engagement with the taboo topic has certain costs. He says: "In broaching a taboo topic, it can be said, individual speakers become subject to a social cost. Whilst this cost is primarily intangible, in the form of these adverse reactions and the 'souring' of an environment, it can also have real, concrete ramifications, be it the exclusion from certain groups or social spaces, a straining of relations, or even, in extreme cases, physical harm" (Crerar 2016, p.199). The antidote to this form of hermeneutic injustice, according to Crerar, is to develop an expressively free environment. Gaile Pohlhaus, Jr., also helpfully expands on the notion of hermeneutic injustice through the notion of willful hermenutical ignorance, which is understood as "instances where marginally situated knowers actively resist epistemic domination through interaction with other resistant knowers, while dominantly situated knowers nonetheless continue to misunderstand and misinterpret the world" (Pohlhaus 2012). This suggests that in order to cultivate expressively free environments, we must develop awareness of how our situated natures and how social situations impact our own abilities and openness to understanding.

These broader conceptions of hermeneutic injustice point to ways in which patience is unevenly distributed because of the various silencing that occurs across communities. Specifically, with respect to making dominant groups aware of oppression, the burden is vastly and disproportionately placed on those who are oppressed to explain their experiences in ways that the nonoppressed individual understands. In addition, those facing oppression frequently face consequences of the kind outlined by Crerar above-straining of relations, further exclusion, and also the greater utilization of cognitive and affective resources in deciding how and when to engage in deliberation.

\section{Cultivating patience to repair argumentative situations}

The patient arguer is one who would take the time to cultivate awareness of these injustices in order to foster expressively free environments and create a better argumentative landscape where 
more people can engage fruitfully. In the section above, I focused on two forms of epistemic injustice: testimonial and hermeneutical injustice, as well as argumentative injustice to motivate my concern that in our non-ideal argumentative world, those who are the most vulnerable to being excluded from arguments, silenced, disbelieved, etc. are the ones who are required to be the most patient. Simultaneously, the more dominantly situated individuals ask them to do the work to make the case that we should care/listen/engage/understand. So, if I am right that patience is an argumentative virtue, then the question becomes, how do we more evenly distribute the burdens of patience in real argumentative situations?

One part of the answer might simply be to accept that patience is an argumentative virtue - one that allows us to appreciate the extended nature of argumentation. For those of us who value argument as an important form of discourse, we can recognize that patience is required in order for argumentation to continue (as opposed to devolving into other forms of settling disagreement) and is also necessary to cultivate more hospitable argumentative environments. It's important to keep in mind here that virtues are context-sensitive, which means that patience will not look the same in every argumentative situation or for every person. As I have argued throughout the paper, it is particularly important to cultivate for people - often those of considerable privilege - who are less aware of how identity, power dynamics, and experience shape argumentative and communicative situations more broadly. Given that virtues are character traits that we can continue to refine and develop, the flexibility of virtue and the sensitivity of virtue to context are useful to help us keep adapting to new circumstances and continue to grow and develop as virtuous arguers. At the same time, virtue argumentation is open to criticism that it does not provide the necessary guidance that is required for particular arguers or argumentative situations. What follows are some suggestions about what strategies could be used broadly to develop the virtue of patience.

In order to develop the argumentative virtue of patience, the arguer should be attentive to the descriptive realities of the argumentative situation rather than just the normative dimensions alone. 
This involves appreciating our messy, non-ideal world rife with injustice and taking the time to try to understand different histories and experiences rather than just engaging them without the preliminary work of assessing our own limitations. The patient arguer must be conscious of her particular history, biases, contexts, framework propositions, and so on, while also striving to understand these aspects of her interlocutors.

A related strategy for developing the virtue of patience might involve adopting more pluralistic and inclusive conceptions of argument. As Patricia Hill Collins tells us, "[t]raditionally, the suppression of Black women's ideas within White-male-controlled social institutions led African-American women to use music, literature, daily conversations, and everyday behavior as important locations for constructing a Black feminist consciousness" (Collins 2008). Exclusion of these ways of knowing is damaging to all of us as knowers and interlocutors, and a more expansive understanding of argumentation and ways of knowing is necessary for inclusive, effective argumentation. Relatedly, Tempest Henning has recently made a compelling argument that the Non-Adversarial Feminist Model of Argumentation primarily prizes the communicative style of white women, and the blanket critique of adversarial discourse excludes the kind of productive adversariality we can see in exchanges in African American women's speech communities that developed in opposition to oppression (Henning 2018). This suggests that blanket critiques of modes of discourse as nonargumentative, or problematically argumentative, should be approached with greater care.

Kristie Dotson has made forceful calls for a more inclusive and pluralistic practice in the academic discipline of philosophy in response to Anita Allen's challenge to philosophy to show what it has to offer Black women (Dotson 2012). Dotson's aptly named "How is this paper philosophy?" is organized around her concern about a "disciplinary culture that renders such a question of paramount importance" (Dotson 2012, p. 5). The question "how is this philosophy" arises, according to Dotson, because the academic discipline of philosophy is a culture of justification: 
a culture that privileges legitimation according to presumed commonly-held, univocally relevant justifying norms, which serves to amplify already existing practices of exceptionalism and senses of incongruence within the profession. Ultimately, I claim that the environment of professional philosophy, particularly in the U.S., bears symptoms of a culture of justification, which creates a difficult working environment for many diverse practitioners. I agree with Allen's assessment that professional philosophy is simply not an attractive setting for many diverse practitioners (Dotson 2012).

Dotson goes on to argue that in order to answer Allen's challenge, philosophy must shift from a culture of justification to a culture of praxis. A culture of praxis both values lived experience to identify live problems and recognizes multiple canons and methodologies. The aim, then, is to "create an environment where incongruence becomes a site of creativity for ever-expanding ways of doing professional philosophy" (Dotson 2012, p. 17). These lessons apply to the broader domain of argumentation as well.

Finally, we might cultivate more patience through consideration of the modes of argumentation. Michael Gilbert has been arguing across his career for a multimodal model of argumentation that extends beyond the logical mode to the emotional, visceral (physical), and kisceral (intuitive) (Gilbert 1994). He emphasizes that different arguments have different primary forms, and to reduce elements such as the emotional to the logical changes the argument. Beyond just the change in meaning that such a reduction would entail, according to Gilbert, "The main point, though, is that this particular story allows us to consider more of the human facets involved in argument," which should be "grist for the argumentation theorist's mill" (Gilbert 1994, p. 175). These moves towards more complicated models of argumentation may be the sign of patient arguers and the kind of arguers we ought to aspire to be.

\section{Acknowledgements}

Thank you to the organizers of the OSSA 2020 virtual conference, where I was able to present an earlier version of this paper, for such rich and interesting discussions. Many thanks to Tracy Bowell for her thoughtful commentary during the conference (and for 
waking up so early so that I could present on Wednesday afternoon from Rochester, NY, while she simultaneously gave her commentary early Thursday morning from New Zealand!) and subsequent comments as well. Thank you to Claudio Duran for making me think more deeply about deep disagreement and to an anonymous reviewer for their thoughtful discussion. Finally, thank you to my writing group at the University of Rochester for their friendship and support and to Alicia Chester for being an early and supportive reader.

\section{References}

Aberdein, Andrew. 2010. Virtue in argument. Argumentation; Dordrecht 24(2):165-179.

- 2016. The vices of argument. Topoi 35(2): 413-422.

2019. Courageous arguments and deep disagreements. Topoi. $<$ https://doi.org/10.1007/s11245-019-09679-w $>$.

Aikin, Scott F. 2019. Deep disagreement, the dark enlightenment, and the rhetoric of the red pill. Journal of Applied Philosophy 36(3): 420435 .

. 2020. What optimistic responses to deep disagreement get right (and wrong). Co-Herencia 17(32): 225-238.

Aikin, Scott F. and J. Caleb Clanton. 2010. Developing groupdeliberative virtues. Journal of Applied Philosophy 27(4): 409-24.

Amenábar, Diego Castro. 2018. Persistent disagreement and argumentation: A normative outline. In ISSA Conference

$<$ https://www.researchgate.net/publication/332274881_Persistent_dis agreement_and_argumentation_A_normative_outline $>$.

Bondy, Patrick. 2010. Argumentative injustice. Informal Logic 30(3): 263-278. <https://doi.org/10.22329/il.v30i3.3034>.

Bowell, Tracy and Justine Kingsbury. 2013. Virtue and argument: Taking character into account. Informal Logic 33(1): 22-32.

Callan, Eamonn. 1993. Patience and courage. Philosophy 68(266): 523539.

Callard, Agnes. 2018. Aspiration: The agency of becoming. New York: Oxford University Press.

Campolo, Chris. 2019. On staying in character: Virtue and the possibility of deep disagreement. Topoi 38(4): 719-23.

Cappelen, Herman and Josh Dever. 2019. Bad Language. New York: Oxford University Press. 
Cohen, Daniel H. 2005. Arguments that backfire. In Argument and its uses, ed. David Hitchcock, 58-65. Hamilton, ON: OSSA.

Cohen, Daniel H. and George Miller. 2016. What virtue argumentation theory misses: The case of compathetic argumentation. Topoi 35(2): 451-60.

Collins, Patricia Hill. 2008. Black feminist thought: Knowledge, consciousness, and the politics of empowerment. 1st edition. New York: Routledge.

Crerar, Charlie. 2016. Taboo, hermeneutical injustice, and expressively free environments. Episteme 13(2): 195-207.

Davson-Galle, Peter. 1992. Arguing, arguments, and deep disagreements. Informal Logic 14(2): 147-156. $<$ https://doi.org/10.22329/i1.v14i2.2537>.

Dotson, Kristie. 2012. How is this paper philosophy? Comparative Philosophy: An International Journal of Constructive Engagement of Distinct Approaches toward World Philosophy 3(1): 3-29. $<$ https://doi.org/10.31979/2151-6014(2012).030105>.

Duran, Claudio. 2016. Levels of depth in deep disagreement. OSSA Conference Archive 9.

Enoch, David. 2014. Précis of taking morality seriously. Philos Stud 168(3): 819-821.

Fausset, R. 2021, Feb. 24. What We Know About the Shooting Death of Ahmaud Arbery. The New York Times.

$<$ https://www.nytimes.com/article/ahmaud-arbery-shootinggeorgia.html>.

Feldman, Richard. 2005. Deep disagreement, rational Richard resolutions, and critical thinking. Informal Logic 25(1): 13-23. $<$ https://doi.org/10.22329/il.v25i1.1041>.

Fogelin, Robert. 1985. The logic of deep disagreements. Informal Logic. 7(1): 1-8. <https://doi.org/10.22329/il.v7i1.2696>.

Fricker, Miranda. 2007. Epistemic injustice: power and the ethics of knowing. Oxford, New York: Oxford University Press.

Gilbert, Michael A. 1994. Multi-modal argumentation. Philosophy of the Social Sciences 24(2): 159-177.

Henning, Tempest. 2018. Bringing wreck. Symposion. URL accessed 10 January 2020: <www.pdenet.org/pdc/bvdb.nsf>.

Kawall, Jason. 2016. Patience. In International Encyclopedia of Ethics. Oxford, UK: John Wiley \& Sons, Ltd.

Kjeldsen, Jens. 2020. What makes us change our minds in our everyday life? Working through evidence and persuasion, events and experiences. OSSA Conference Archive.

$<$ https://scholar.uwindsor.ca/ossaarchive/OSSA12/Saturday/7.>. 
Kloster, Moira. 2018. Another dimension to deep disagreements: Trust in argumentation. Topoi. <https://doi.org/10.1007/s11245-018-9617$6>$.

Lemire, Jonathan, Darlene Superville, Will Weissert and Michelle L. Price. 2020. Chaotic first debate: Taunts overpower Trump, Biden visions. URL accessed 30 September 2020: <https://apnews.com>.

Lugg, Andrew. 1986. Deep disagreement and informal logic: No cause for alarm [Reply]. Informal Logic. 8(1): 47-51. $<$ https://doi.org/10.22329/il.v8i1.2680>.

McKinnon, Rachel. 2015. Trans*formative experiences. Res Phil 92(2): 419-440.

Paul, L. A. 2015. Précis of Transformative experience. Philosophy and Phenomenological Research 91(3): 760-65.

Pohlhaus, Gaile. 2012. Relational knowing and epistemic injustice: Toward a theory of "willful hermeneutical ignorance." Hypatia 27(4): 715-35.

Vigani, Denise. 2017. Is patience a virtue? J Value Inquiry 51(2): 327 40.

Woodward, C. and Riechmann, D. 2021, January 9. No surprise: Trump left many clues he wouldn't go quietly. AP News. $<$ https://apnews.com/article/donald-trump-ap-top-news-conspiracytheories-elections-828feb5006a2fad60f457f5a6cbe731f $>$. 